\title{
MUTAGÉNÈSE AU NIVEAU GÉNIQUE
}

\author{
Ethel MOUSTACCHI \\ Institut Curie-Biologie, URA 1292 du CNRS \\ 26 rue d'Ulm, 75231 Paris cedex 05
}

La caractérisation des altérations moléculaires présentes dans les mutants "spontanés" apparaissant in vivo chez l'homme est indispensable si l'on souhaite établir le spectre des mutations dues à un agent physique ou chimique spécifique. L'espoir est que, pour certains mutagènes au moins, une "signature moléculaire" caractéristique de l'exposition à cet agent sera reconnaissable. Les mesures de mutagénicité chez l'homme fourniraient alors une évaluation à la fois qualitative et quantitative de la génotoxicité complétant les données de clastogénèse.

Le spectre des modifications "spontanées" produites in vivo au niveau de l'ADN des lymphocytes $\mathrm{T}$ circulants chez l'homme a été défini pour le gène de l'hypoxanthine-guanine phosphoribosyl transferase (HPRT). $15 \%$ de ces mutations sont constituées chez l'adulte par des réarrangements structuraux et des délétions supérieures à 100 paires de bases dont les points de rupture sont distribués au hasard au sein du gène HPRT. Les $85 \%$ des mutations restantes sont des mutations dites "ponctuelles" dues à des changements d'une base (transversions et transitions), des petites insertions ou délétions, des mutations de glissement du cadre et des changements de sites d'épissage. Ces altérations ponctuelles sont également réparties au hasard dans le gène HPRT (Turner et al., 1985 ; Nicklas et al., 1987, 1989 ; Albertini et al., 1990 ; Davies et al., 1992).

Un autre marqueur génétique d'intérêt pratique est constitué par le gène de la glycophorine A (GPA) dont on peut mesurer la fréquence des variants dans les érythrocytes du sang périphérique (Jensen et al., 1991). Il s'agit d'un gène autosomal qui régule la synthèse d'une sialoglycoprotéine exprimée à la surface des érythrocytes humains (antigènes de surface des groupes sanguins $M$ et $N$ ). Le test GPA permet la mesure par cytofluorimétrie de la perte d'expression de l'une des formes alléliques $M$ ou $N$ due à des mutations survenant dans les précurseurs érythrö̈des. Chez les hétérozygotes $M N$, on énumère la fréquence des variants NO ou MO (qui ont perdu l'expression des allèles $\mathrm{M}$ ou $\mathrm{N}$, respectivement) ou des variants $N N$ ou $M M$ qui ont perdu l'expression d'un allèle et expriment deux copies de l'allèle restant. Le premier type d'évènement (variants dits "nuls") résulte de mutations ponctuelles, de délétions, d'aneuplö̈die ou de mis-ségrégation. Le second type d'évènements est dû à de la recombinaison ou à de la non-disjonction. A 
titre d'exemple, chez les donneurs normaux, la fréquence des variants NO est comprise entre 5 et $13 \times 10^{-6}$ cellules et celle des variants $\mathrm{NN}$ est comprise entre 3 et $12 \times 10^{-6}$ cellules.

Plusieurs laboratoires, dont le nôtre, s'attachent à l'examen des lymphocytes pour le gène $H P R T$ et des érythrocytes pour le marqueur GPA de populations humaines soumises à des irradiations : 1) à des fins thérapeutiques (Sala-Trepat et al., 1990 et références inclues) ou 2) par accident (survivants d'Hiroshima et de Nagasaki) (Langlois et al., 1987 ; Hakoda et al., 1988, 1989 ; Kyoizumi et al., 1989 a ; Akiyama et al., 1990). Une augmentation, en fonction de la dose de radiations, de la fréquence de mutations observées in vivo aux deux loci est clairement observée. Les mutations induites qui prédominent sont dues à d'importantes altérations structurales, essentiellement des délétions (Nicklas et al., 1991), qui seront décrites : celles-ci ne semblent pas être distribuées de manière aléatoire. Une attention particulière a été récemment portée aux effets de radioisotopes incorporés $\left({ }^{125} \mathrm{I}\right.$, ${ }^{131} \mathrm{I}$ ) et au rayonnement $\alpha$ émis par le radon (Clarke \& Southwood, 1989 ; Xiao et al., 1989 ; Nicklas et al., 1990 ; Aghamohammadi et al., 1992).

L'objectif à long terme de ces travaux est de mieux cerner le risque cancérigène lié aux irradiations à l'aide d'une meilleure définition des mutations somatiques. On verra que la comparaison de donneurs sains à des patients atteints de maladies génétiques prédisposant au cancer et associées à une hypersensibilité aux radiations et/ou à des agents chimiques (ataxie télangiectasie, anémie de Fanconi, syndrome de Bloom, xeroderma pigmentosum) (Kyoizumi et al., 1989 b ; Langlois et al., 1989 ; Cole et al., 1992 ; Sala-Trepat et al., 1993) vient également enrichir notre compréhension de la malignité radioinduite.

\section{Références}

- Akiyama M., Kyiozumi S., Hirai Y., Hakoda M., Nakamura N. \& Awa A.A. (1990) Studies on chromosome aberrations and HPRT mutations in lymphocytes and GPA mutation in erythrocytes of atomic bomb survivors. Mutation and the Environment, Part C, Wiley-Liss, pp. 69-80.

- Albertini R.J., Nicklas J.A., O'Neill J.P. \& Robison S.H. (1990) In vivo somatic mutations in humans : Measurement and analysis. Ann. Rev. Genet., 24, 305-326.

- Aghamohammadi S.Z., Morris T., Stevens D.L. \& Thacker J. (1992) Rapid screening for deletion mutations in the hprt gene using the polymerase chain reaction : X-ray and $\alpha$-particle mutant spectra. Mutation Res., 269, 1-7.

- Clarke R.H. \& Southwood T.R.E. (1989) Risks from ionising radiation. Nature, 338, 197-198. 
- Cole J., Arlett C.F., Norris P.G., Stephens G., Waugh A.P.W., Beare D.M. \& Green M.H.L. (1992) Elevated hprt mutant frequency in circulating T-lymphocytes of xeroderma pigmentosum patients. Mutation Res., 273, 171-178.

- Davies M.J., Lovell D.P. \& Anderson D. (1992) Thuiguanine-resistant mutant frequency in T-lymphocytes from a healthy human population. Mutation Res., 265, 165-171.

- Hakoda M., Akiyama M., Kyiozumi S., Awa A.A., Yamakido M. \& Otake M. (1988) Increased somatic cell mutant frequency in atomic bomb survivors. Mutation Res., 201, $39-48$.

- Hakoda M., Hirai Y., Kyoizumi \& Akiyama M. (1989) Molecular analysis of in vivo hprt mutant T cells from atomic bomb survivors. Environ. Mol. Mutagen., 13, 25-33.

- Jensen R.H., Grant S.G., Langlois R.G. \& Bigbee W.L. (1991) Somatic cell genotoxicity at the glycophorin A locus in humans. In : B.L. Gledhill \& F. Mauro (Eds.), New Horizons in Biological Densitometry, Wiley-Liss, Inc,, vol. 372, pp. 329-339.

- Kyoizumi S., Nakamura N., Hakoda M., Awa A.A., Bean M.A., Jensen R.H. \& Akiyama M. (1989 a) Detection of somatic mutations at the glycophorin A locus in erythrocytes of atomic bomb survivors using a single beam flow sorter. Cancer Res., 49, $581-588$.

- Kyoizumi S., Nakamura N., Takebe H., Tatsumi K., German J. \& Akiyama M. (1989 b) Frequency of variant erythrocytes at the glycophorin-A locus in two Bloom's syndrome patients. Mutation Res., 214, 215-222.

- Langlois R.G., Bigbee W.L., Kyoizumi S., Nakamura N., Bean M.A., Akiyama M. \& Jensen R.H. (1987) Evidence for increased somatic cell mutations at the glycophorin A locus in atomic bomb survivors. Science, 236, 445-448.

- Langlois R.G., Bigbee W.L., Jensen R.H. \& German J. (1989) Evidence for increased in vivo mutation and somatic recombination in Bloom's syndrome. Proc. Natl. Acad. Sci. USA, 86, 670-674.

- Nicklas J.A., Hunter T.C., Sullivan L.M., Berman J.K., O'Neill J.P. \& Albertini R.J. (1987) Molecular analysis of in vivo hprt mutations in human T-lymphocytes. I : Studies of low frequency "spontaneous" mutants by Southern blots. Mutagenesis, 2, 341-347.

- Nicklas J.A., Hunter T.C., O'Neill J.P. \& Albertini R.J. (1989) Molecular analysis of in vivo hprt mutations in human T-lymphocytes. III : Longitudinal study of hprt gene structural alterations and T-cell clonal origins. Mutation Res., 215, 147-160.

- Nicklas J.A., Falta M.T., Hunter T.C., O'Neill J.P., Jacobson-Kram D., Williams J. \& Albertini R.J. (1990) Molecular analysis of in vivo hprt mutations in human T-lymphocytes. V : Effects of total body irradiation secondary to radioimmunoglobulin therapy (RIT). Mutagenesis, 5, 416-468.

- Nicklas J.A., O'Neill J.P., Hunter T.C., Falta M.T., Lippert M.J., Jacobson-Kram D., Williams J.R. \& Albertini R.J. (1991) In vivo ionizing irradiations produce deletions in the hprt gene of human T-lymphocytes. Mutation Res., 250, 383-396.

- Sala-Trepat M., Cole J., Green M.H.L., Rigaud O., Vilcoq J.R. \& Moustacchi E. (1990) Genotoxic effects of radiotherapy and chemotherapy on the circulating 
- lymphocytes of breast cancer patients. III : Measurement of mutant frequency to 6-thioguanine resistance. Mutagenesis, 5, 593-598.

- Sala-Trepat M., Boyse J., Richard P., Papadopoulo D. \& Moustacchi E. (1993) Frequencies of $H P R T^{-}$lymphocytes and glycophorin A variants erythrocytes in Fanconi anemia patients, their parents and control donors. Mutation Res. (in press).

- Turner D.R., Morley A.A., Haliandros M., Kutlaca B. \& Sanderson B.J. (1985) In vivo somatic mutations in human lymphocytes frequently result from major gene alterations. Nature, 315, 343-345.

- Xiao S.Q., Jacobson-Kram D., Piantadosi S. \& Williams J.R. (1989) Increased chromosomal radiosensitivity in patients undergoing radioimmunotherapy. Mutation Res., 227, 39-45. 